\title{
An Evolutionary Algorithm of the Regional Collaborative Innovation Based on Complex Network
}

\author{
Kun Wang and Duoyong Sun \\ College of Information System and Management, National University of Defense Technology, Changsha, Hunan 410073, China \\ Correspondence should be addressed to Kun Wang; wangkunt@gmail.com
}

Received 19 May 2015; Revised 22 July 2015; Accepted 15 September 2015

Academic Editor: Carlo Piccardi

Copyright (c) 2016 K. Wang and D. Sun. This is an open access article distributed under the Creative Commons Attribution License, which permits unrestricted use, distribution, and reproduction in any medium, provided the original work is properly cited.

\begin{abstract}
This paper proposed a new perspective to study the evolution of regional collaborative innovation based on complex network theory. The two main conceptions of evolution, "graph with dynamic features" and "network evolution," have been provided in advance. Afterwards, we illustrate the overall architecture and capability model of the regional collaborative innovation system, which contains several elements and participants. Therefore, we can definitely assume that the regional collaborative innovation system could be regarded as a complex network model. In the proposed evolutionary algorithm, we consider that each node in the network could only connect to less than a certain amount of neighbors, and the extreme value is determined by its importance. Through the derivation, we have created a probability density function as the most important constraint and supporting condition of our simulation experiments. Then, a case study was performed to explore the network topology and validate the effectiveness of our algorithm. All the raw datasets were obtained from the official website of the National Bureau of Statistic of China and some other open sources. Finally, some meaningful recommendations were presented to policy makers, especially based on the experimental results and some common conclusions of complex networks.
\end{abstract}

\section{Introduction}

As is illustrated in Melanie Mitchell's famous book named "Complexity: A Guided Tour" [1], an accepted concept of complex networks refers to a graph with nontrivial topological features. These features belonged to complex network, but they rarely occur in simple networks (e.g., lattices or random graphs). And they do often appear over and over in the real world. Since the two landmark achievements of Professors Watts and Strogatz [2] and Professors Barabási and Albert [3] were published, respectively, in the journal Nature in 1998 and in the journal Science in 1999, network science has attracted more and more attention [4]. It also becomes a hot topic in both computer networks and social networks. The recent research field of complex networks has expanded to the networks of networks [5]. However, although complex network is of great importance in many research fields, the scholars have not figured out its internal structure and properties in complete detail. Randomness is considered to be the major features of complex networks in the traditional study, but it was overturned in last year of the twentieth century. And it had exerted remarkable influence on understanding how the complex networks are constructed and worked [6]. Particularly, the appropriate evolution model should not only obtain the processes that assembled networks correctly and completely but also make it clear to what extent the microscopic processes affect the network topology [7].

Considering that complex network may change its internal structure with time varying, hence, it becomes quite meaningful to intensely research the dynamic behaviors of complex network. However, previous research of complex network evolution models mainly concentrates on the macroscopic properties such as the Small-World effect and Scalefree property $[8,9]$. If we only consider the macroscopic features, it might be difficult to provide credible review among different kinds of evolution models [10]. Therefore, local structures and an excellent model of evolution should be further studied in the future.

At the same time, regional collaborative innovation has been deeply studied on both theory and practice. For some 
time, many local governments attempt to construct regional cooperation organizations in order to inspire the innovation enthusiasm of different innovators and enterprises [11]. Meanwhile, regional collaborative innovation systems have also played important and positive roles in enhancing the comprehensive innovative capacity and the level of economic development [12]. That is, it is of great importance to promote the regional economy through translation of innovation into products and services. Particularly, researching and revealing the evolution rules of the innovative organizations from complex network perspective can help government to understand their laws and characteristics better and foster new growth points of local economic development [13]. Furthermore, the policy makers might also benefit from the conclusions of this paper.

Our paper has proposed a novel method to analyze the regional collaborative innovation system by applying the evolutionary theory of complex network. And the rest of the paper is structured as follows. Section 2 presented some relevant literatures. In Section 3, we explained how to obtain proper understanding of the evolution of regional collaborative innovation systems. Section 4 has given a detailed introduction of the evolutionary algorithm to analyze the above systems from complex network perspective. To verify the effectiveness of our proposed algorithm, a case study is designed and conducted in Section 5. And some recommendations have also been presented in this part. Finally, conclusions are drawn in Section 6.

\section{Related Works}

The conception of complex network has been widely introduced in many fields of both the nature and social science. As is well known, complex networks are made up of a multitude of interacting nodes communicating with others based on the interconnections of complex networks [14]. Meanwhile, complex network provides an effective method to describe many application systems and contain the following attributes: (1) topology, (2) dynamics, and (3) evolution. In this paper, we would focus solely on the evolution of complex network, and some relevant literatures are listed as follows. The paper list is sorted according to their main research contribution of the network evolution, such as the architectural attributes, the related applications, the evaluation methods, and the evolution models.

Kasthurirathna et al. focused on the problem how the topological structures of a social system can influence the network evolution. In this paper, the authors simulated a coordination game on approximately four different classes of complex networks which have been commonly utilized to construct the social systems. Experimental results show that the time-lags and noise in information about relative payoffs could continuously influence the emergence of coordination mechanisms [15].

Anzo and Barajas-Ramirez studied the influences of the structural evolution on stability of the synchronized behavior in the complex network system. The main innovations of this paper lie in that the structural evolution used in this paper is divided into classes: that is, (1) the topology changes with an arbitrary switching law among a set of admissible patterns of connection and (2) the strength of connection that evolves is determined by an adaptive law [16].

Alvarez-Martinez et al. utilized the complex network evolution technology to predict the birth and death ratio. In this paper, master equations for the evolution of complex networks with birth and negative transition probabilities per unit time are analyzed in detail. Particularly, the basic dynamical function for its stationary solution refers to the ratio between drift and diffusion coefficients [17].

In the field of information processing in the smart grid, Pagani and Aiello studied how different network topologies and growth models could accelerate a more efficient and reliable network and how a power company can facilitate integration of decentralized electricity market. Furthermore, this paper has illustrated the importance of network connectivity in enhancing the properties of reliability and path-cost reduction. Finally, empirical results indicate that such evolution method creates a better balance between the sustained growth in connectivity and the cost of network size increase [18].

The Linux kernel is a good example of the most popular Open Source Operating Systems. In [19], the authors focused on the application of network evolution in information science and proposed an entirely new way to study the evolution of the Linux kernel components based on the complex network theory. After investigating the node degree distribution, clustering coefficient, hierarchical structure, and average path length of the Linux kernel from version 1.0 to version 2.4.35, the authors found that, in the system, NET components, file systems, memory management, and the underlying driver are all showing more or less the SmallWorld effect and Scale-free property [19].

Duan and Zhan concentrated on the evolution of node importance in a network through the information of cascading failures. On the one hand, a new indicator of node importance is presented in this paper, according to the load turbulence of each node. On the other hand, the evolution of its node importance can be implemented with the load redistribution rule, node capacity, and network topology [20].

Huang et al. presented the conception and evaluation indexes of the emergency logistics network connection reliability to construct the evaluation index system of complex network and then illustrated the proposed indexes quantitatively to test the reliability of existing network connections. Furthermore, the authors proposed a novel network topological model and develop simulation tools to test the reliability of the system when attacking behavior happens [21].

Leskovec et al. raised several interesting questions at the beginning of their widely cited essay about the graph evolution as follows. "What are normal growth patterns in social, technological, and information networks?" "How do real graphs evolve over time?" Through reviewing the relevant studies about degree power laws, which show that the set of node degrees has a heavy-tailed distribution, the authors pointed out the defect of existing graph generation models. After comparing with many previous empirical studies of the large-scale networks, they provided a new graph generation algorithm, based on the Forest Fire spreading process [22]. 
Hadzibeganovic et al. proposed a novel agent-based evolutionary model of the tag-mediated altruism, which is suitable in large-scale complex networks. Being different from the former theoretical predictions, authors found that altruistic acts in nonrepeated interactions could emerge as a natural consequence to recognize heritable phenotypic traits such as visual tags. Furthermore, authors discovered the topological regimes in which cooperation usually prevails in a short period of time [23].

From the above related works, we could see that the complex system evolution has heated up in many fields over the past few years. As we all know, the unique feature of the complex networks is the Anticategorical Imperatives [24]. And it has been validated over and over again in the largescale networks, which contains a large amount of nodes of the same type. However, according to the literatures, the evolution method of complex system has not been widely used in the research of regional collaborative innovation. Hence, in this paper, we will provide an efficient algorithm to evaluate the evolution of the regional collaborative innovation system based on the complex network theory.

\section{Explanation for Complex Network Evolution}

Before providing our evolutionary algorithm to analyze the regional collaborative innovation based on the complex network, the main problem, how a complex network evolution can be completed, should be explained at first, and it contains four main parts as follows:

(1) $\Theta$ : a set of possible structures in the complex system,

(2) $X$ : a set of input elements from the outside environment,

(3) $\Psi$ : a set of operators which are utilized to improve organizational structures in the complex system,

(4) $\Gamma: X \times \Theta \rightarrow \Psi$ : adaptive control plan.

It is well known that complex network can be represented as a generalized dynamic graph $G=(V, E)$, in which $V=$ $\left\{v_{1}, v_{2}, \ldots, v_{n}\right\}$ and $E=\left\{e_{i j}\right\}, i, j \in\{1,2, \ldots, n\}$ represent the node set and the edge set, respectively. In order to explain the process of network evolution more intuitively, we provide a brief example in Figure 1.

As is shown in Figure 1, the evolving forms of a specific network fall broadly into three categories. In this figure, the node importance is represented by the radius of the node, and the edge weight is denoted by the thickness of the line. That is, the bigger the node radius is, the more important the node is. Meanwhile, the thicker the line is, the greater the edge weight is. In state 1 , an original graph with six nodes and seven edges is included. Particularly, with the network dynamics varying, the node importance and edge weight are changed as well. Afterwards, state 1 is transformed to state 2 by modifying their current connectivity and the edge weights. Then state 2 is converted to state 3 through adding some new nodes and edges. Afterwards, the two important concepts in network evolution are defined as follows.
Definition 1 (graph with dynamic features). For a given graph with $N$ nodes, the network structure and the operators are represented as $\mu$ and $\nu$, respectively. Hence, the dynamic graph can be represented as $\mathrm{DG}=(N, V, \mu, E, U, T, \nu)$, in which symbols are defined as follows.

(1) $N=\left\{n_{1}, n_{2}, \ldots, n_{m}\right\}$ is the finite set of graph nodes.

(2) $V=\left\{v_{1}, v_{2}, \ldots, v_{m}\right\}$, among which $v_{i}$ refers to the set of node states.

(3) $\mu=\left\{a_{1}, a_{2}, \ldots, a_{n}\right\}$ and $a_{i} \in V \times V$ means the finite set of graph edges.

(4) $E=\left\{e_{1}, e_{2}, \ldots, e_{m}\right\}$, among which $e_{i}$ refers to the set of edge states.

(5) $U=\left\{u_{1}, u_{2}, \ldots, u_{p}\right\}$, where $u_{i}$ is the set of external input elements.

(6) $T$ means the set of times which is related to network dynamics.

(7) $\Pi: V \times E \times U \times T \rightarrow V \times E$ denotes converting between different node or edge states.

Definition 2 (network evolution). Utilizing the graph recommended in Definition 1, the process of network evolution can be summarized as the collection $\Upsilon=(D, \Psi, \Xi, P)$, and symbols in $\Upsilon$ are defined as follows.

(1) $D=\left(d_{1}, d_{2}, \ldots, d_{x}\right)$ represents the set of dynamic graphs.

(2) $\Psi=\left\{\eta_{1}, \eta_{2}, \ldots, \eta_{y}\right\}$ denotes the set of structural operators.

(3) $\Xi$ is input from outside environment.

(4) $P: \Xi \times D \rightarrow \Psi$ refers to evolution plan which could choose the operators $\eta \in \Psi$ to be utilized when the current structure is converted to a new one.

\section{Understanding the Architecture and Evolution of Regional Collaborative Innovation}

4.1. Structure Model of the Regional Collaborative Innovation. In this section, we will describe the network framework of regional collaborative innovation and discuss why complex network evolution can analyze these systems effectively. Firstly, in Figure 2, the basic elements of the regional collaborative innovation are illustrated, in which case, the foundation of innovation resources should be supported by both the hard environment and soft environment. Afterwards, the innovation, the transformation, and the achievement of knowledge could be circulated in the regional collaborative innovation system.

Secondly, in order to study the whole organizational structure more profoundly and meticulously, as is shown in Figure 3, the structure of the regional innovation system in detail from the collaborative perspective has been described in Figure 3.

The concept of complex networks is commonly represented by a medium number of interactions with patterns 


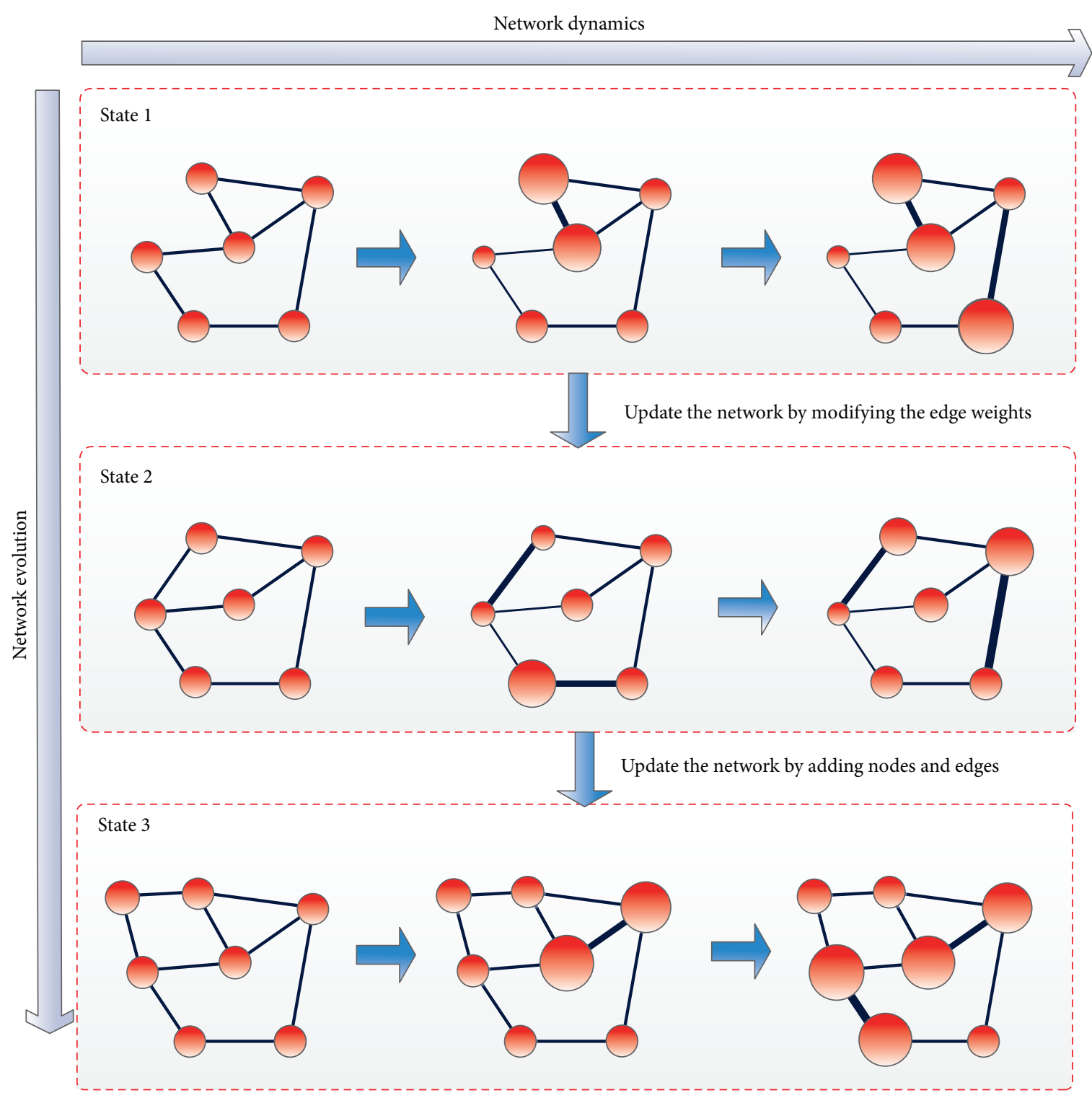

FIGURE 1: Illustration of the network evolution.

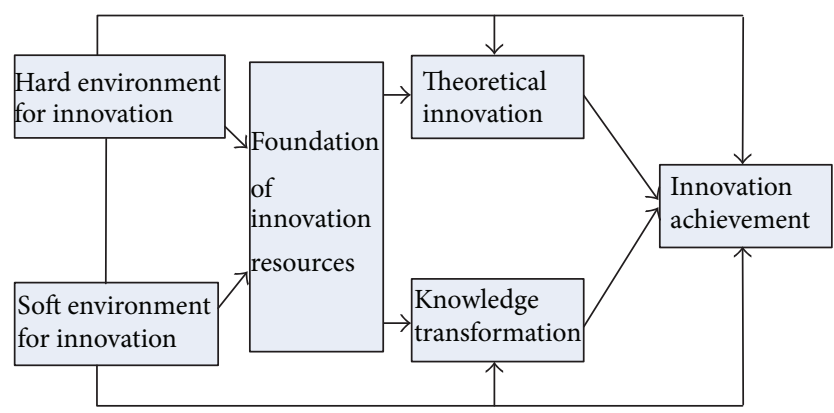

FIgURE 2: The basic elements of the regional collaborative innovation.

of elements among them, such as the social, biological, and technological networks [25]. As illustrated in Figures 2 and
3, the regional collaborative innovation system could also be regarded as a complex system, in which several participants are included, such as the government, intermediary organizations, universities, enterprises, research institutes, and financial institutions. Meanwhile, Figure 3 shows that the collaborative innovation collaborative system can run well only when the relationships among the different participants and elements could coordinate with each other effectively. And the most important part is to communicate and exchange in whatever form or manifestation.

4.2. Algorithm Description. In order to analyze the evolution of regional collaborative innovation system more effectively, an evolutionary algorithm will be introduced in this section. Considering the situation that excessive amounts of edges in the complex network may low down the overall performance of the system, in our proposed algorithm, we have added 


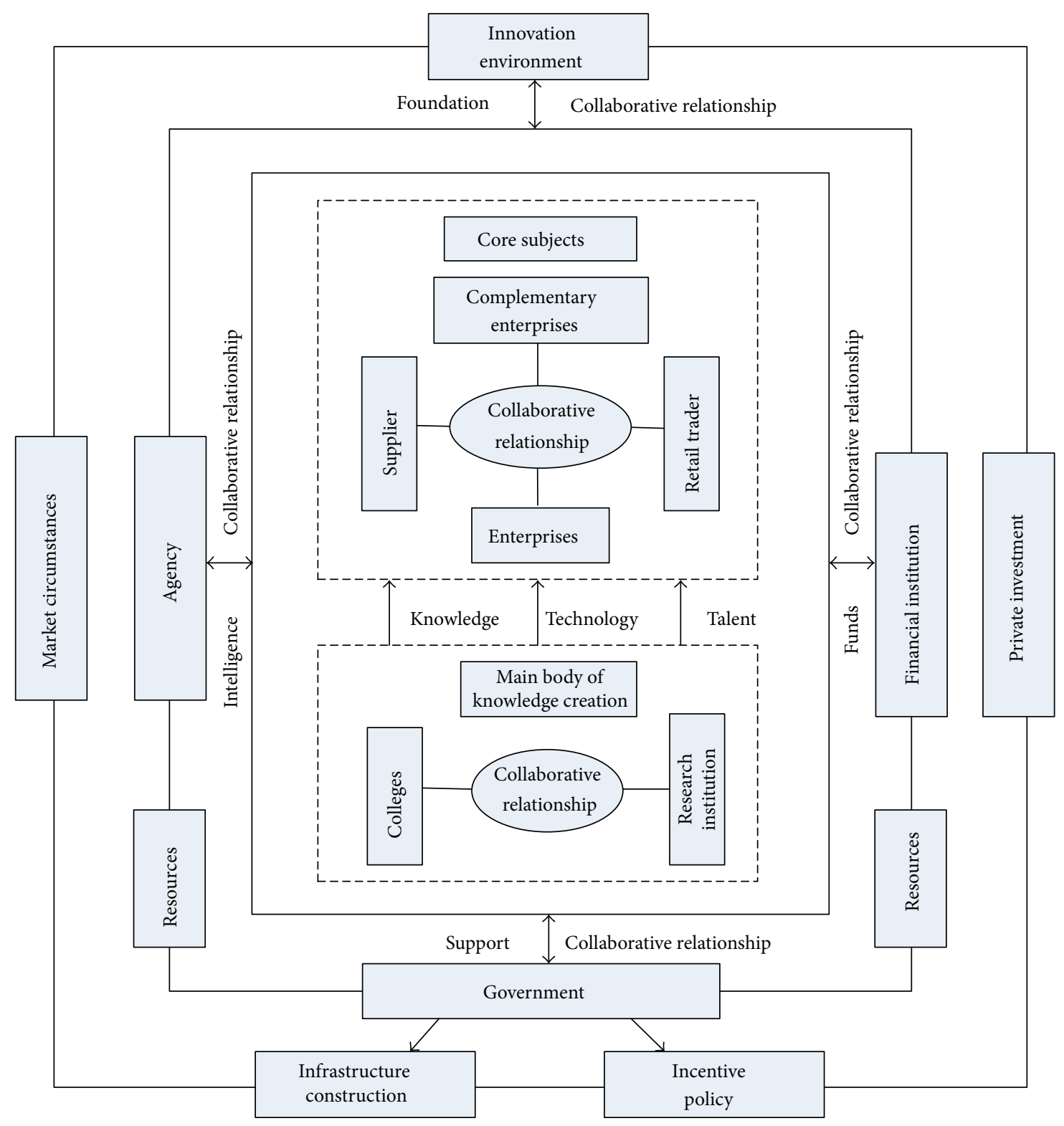

FIGURE 3: Architecture of the regional innovation system under the collaborative perspective.

some constraint conditions. The main idea of our algorithm are based on the basic fact that we assume each node in the complex network can connect to less than a specific number of neighbors. For a certain node, the maximum number of its neighbors is mainly determined by the nodal weight and its connectivity. And the algorithm will be described in detail as follows.

Step 1. Start with $N$ initial nodes, and then construct a fully connected graph.

Step 2. At each time slot, choose an existing node with uniform probability, and then delete amount of edges which currently connect the node. And the number is primarily based on the node importance and must be less than a threshold. In a general sense, we choose $N / 8$ as the ceiling of this step.

Step 3. For a newly added node, select the nodes that already exist in the network, and determine which ones the new node should connect. For an existing node $i$, probability $P_{i}$, whether it would be selected by the new node or not, is influenced by its nodal weight $\left(I_{i}\right)$ and node connectivity $\left(C_{i}\right)$. And $P_{i}$ can be computed as follows:

$$
P_{i}=\frac{f\left(I_{i}, C_{i}\right) \cdot C_{i}}{\sum_{j \in \text { Neighbors of node } i} f\left(I_{j}, C_{j}\right) \cdot C_{j}},
$$

where $f\left(I_{i}, C_{i}\right)$ represents the probability of the existing node $i$ to connect the newly added nodes which is influenced by its 
nodal weight and node connectivity. That is, when $C_{i}$ is closer to its extreme value, the selected node $i$ might be improbable to connect with other new nodes.

Step 4. According to the mean-field theory, the following equation should be used:

$$
\frac{\partial\left(C_{i}\right)}{\partial(t)}=\frac{m \cdot f\left(I_{i}, C_{i}\right) \cdot C_{i}}{\sum_{j \in \text { Neighbors of node } i} f\left(I_{j}, C_{j}\right) \cdot C_{j}},
$$

where $m$ denotes the number of edges which are finalized to connect the newly added nodes. As to the total number of node in the network, the following equation is satisfied:

$$
\sum_{j \in \text { Neighbors of node } i} f\left(I_{j}\right) \cdot C_{j}=R \cdot \widehat{I} \cdot \operatorname{AVG}(D),
$$

where $R$ denotes the number of neighborhoods of the recently added node, $\widehat{I}$ refers to the expected value of function $f(I)$, and $\operatorname{AVG}(D)$ means the average degree of the whole network. $\operatorname{AVG}(D)$ can be calculated as follows:

$$
\operatorname{AVG}(D)=\frac{2 q t+q_{0}}{r_{0}+t}
$$

where $q_{0}$ and $r_{0}$ mean the number of edges and the number of nodes at initial state, respectively. Symbol $q$ denotes the number of edges of the newly added node.

Step 5. For the given node $i$, its connectivity $\left(C_{i}\right)$ must be restricted below a threshold, because of the limitations of time and resource on the real situation:

$$
C_{i}=\frac{\max \left(C_{i}\right)}{1+\left(\max \left(C_{i}\right)-q\right) / q \cdot e^{I /(R \cdot \widehat{I}) \cdot\left(t_{i}-t\right)}} .
$$

Step 6. In order to achieve the probability density function, the following equation should be calculated:

$$
\begin{aligned}
P(C)= & \int_{I_{\min }}^{I_{\max }} \delta \cdot \frac{2 \cdot R \cdot \widehat{I}}{q_{0}+t} \cdot \frac{C_{\max }}{I_{\max }} \\
& \cdot \frac{1}{C \cdot \max \left(C_{i}\right)-C^{2}} d I,
\end{aligned}
$$

where parameter $\delta$ refers to the distribution of node importance $I$ and $C$ means the continuous random variable to represent the node connectivity. $I_{\max }$ and $I_{\min }$ represent the maximum and minimum value of the nodal weight in the network.

\section{Case Study and Results Analyzing}

Data collection is a key part during the experiment, so how to obtain data source is very important. The collected dataset must be from the official way to ensure the authenticity. Hence, the credibility of empirical findings can be guaranteed only when the dataset is accurate. This study requires a lot of raw data to construct the initial models of regional collaborative innovation from complex system perspective. Therefore, we got most of raw dataset through the official website of the National Bureau of Statistic of China (NBS) and some other open sources: for example, (1) comprehensive statistical yearbook of the country, (2) comprehensive statistical yearbook issued by local governments, (3) professional statistical yearbooks of government departments, and (4) other kinds of publications.

More specifically, many kinds of related publications are referred to, such as the "China Statistical Yearbook," "China Statistical Yearbook on Science and Technology," "China's Regional Economic Statistical Yearbook," "China Statistics Yearbook on High Technology Industry," "Almanac of China's Finance and Banking," "China's Transportation Statistical Yearbook," "Chinese Regional Innovation Capability Report," "The Statistical Analysis of Paper in Chinese Science and Technology," and "Chinese Torch Program Statistics" [26]. However, some missing data were collected directly through some reports released by the relevant government authorities.

As is shown in Figure 4, the internal structure of regional collaborative innovation system is translated into a hierarchical structure which is made up of three levels. At the first level, five main components are included, which are (1) innovation guaranteed ability, (2) innovation research and development ability, (3) innovation integration ability, (4) innovation transformation ability, and (5) innovation supporting environment. These five parts can construct a complete regional collaborative innovation system, and they were very similar to the essential elements, which were shown in Figures 2 and 3. At the same time, it also was considered to be an important basis of the initial weight of nodes in the complex network models. In order to study the evolution of the entire network of regional collaborative innovation, the relevant data from 2008 to 2013 has been collected.

Based on the above raw data, a complex network model of regional collaborative innovation is constructed at first. There are about 953 nodes (individuals) included in the simulation experiments in the initial state. Then, the average payoff and individual cooperation rate of the case are given which are based on the proposed algorithm.

Combining the experimental results in Figures 5 and 6, it can be seen when the number of iterations is larger than 500 , both the average payoff and the individual cooperation rate of the proposed complex network have become stable. As mentioned in the algorithm description, the node connectivity is a core part of the node importance. It is easy to understand the trend of the two curves in Figures 5 and 6. It is what we called the law of diminishing marginal utility [27] in the research of economics. And the famous Limits to Growth model [28] might once again be an excellent verification in the evolution of regional collaborative innovation system.

Afterwards, in Figure 7, we try to test the degree distribution of the complex network, and symbol $K$ denotes the degree of a randomly chosen node. Furthermore, the curve in Figure 7, which presents the degree distribution of complex network, was something similar to following the Power Law Distribution. Moreover, we would expect that the structure of the proposed complex network has been 


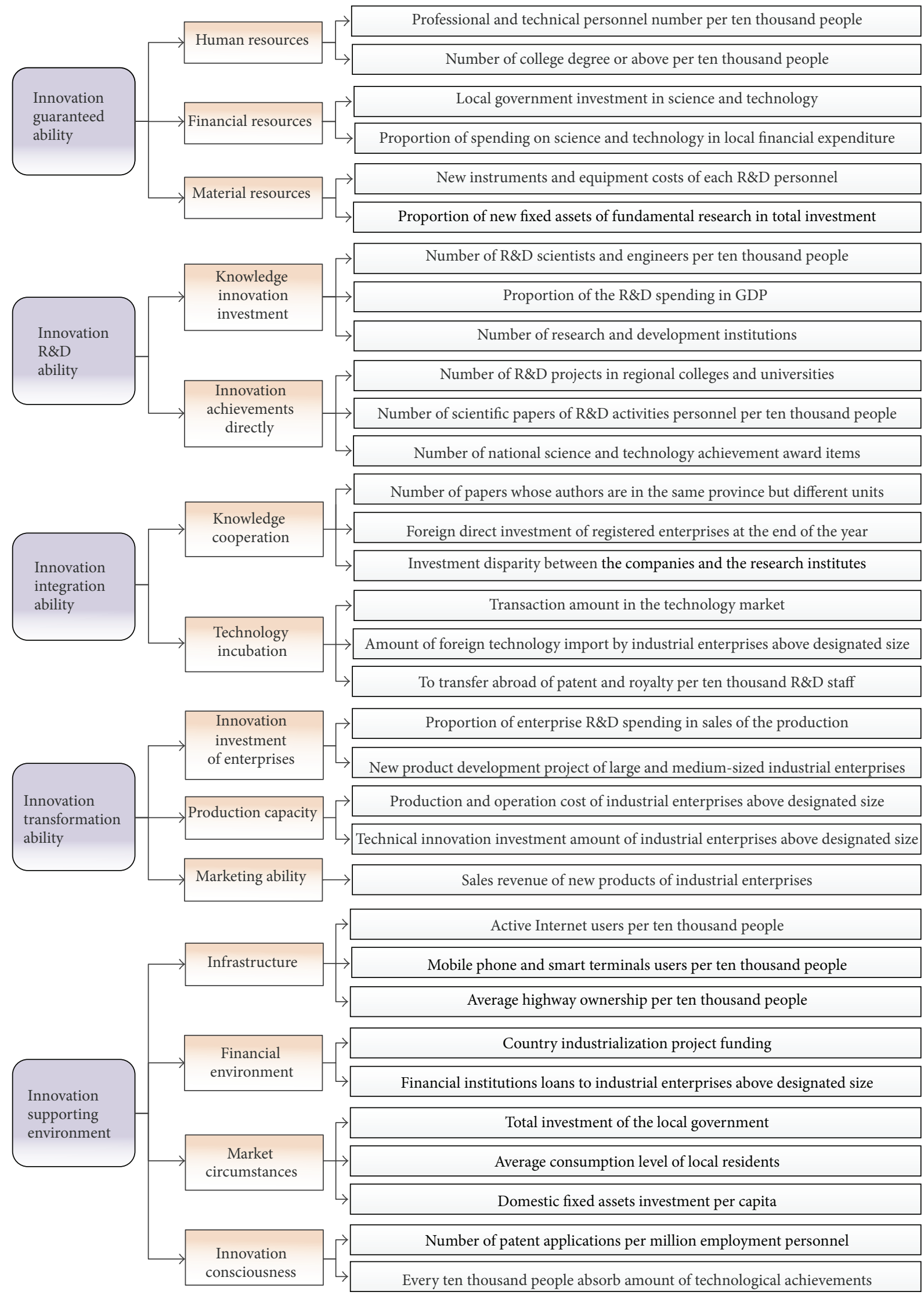

FIGURE 4: The source data used in the analyzing of regional collaborative innovation. 


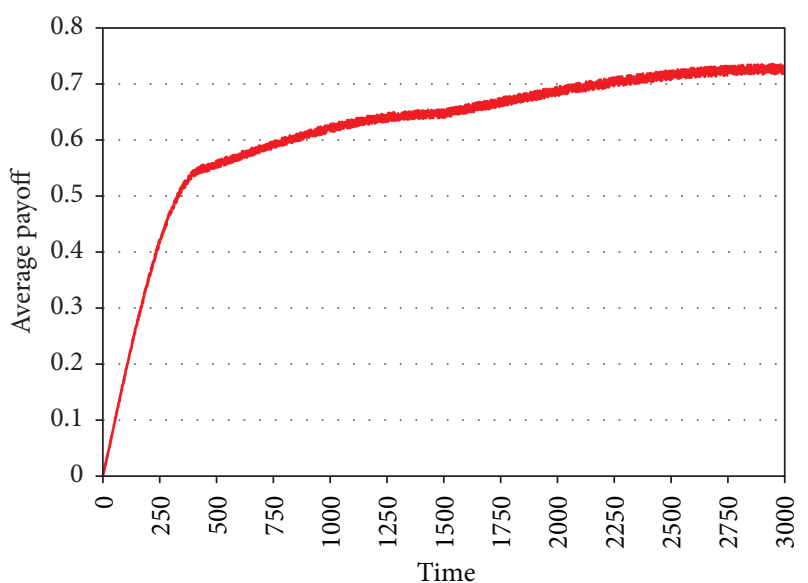

Figure 5: Average payoff of individuals of the proposed complex network.

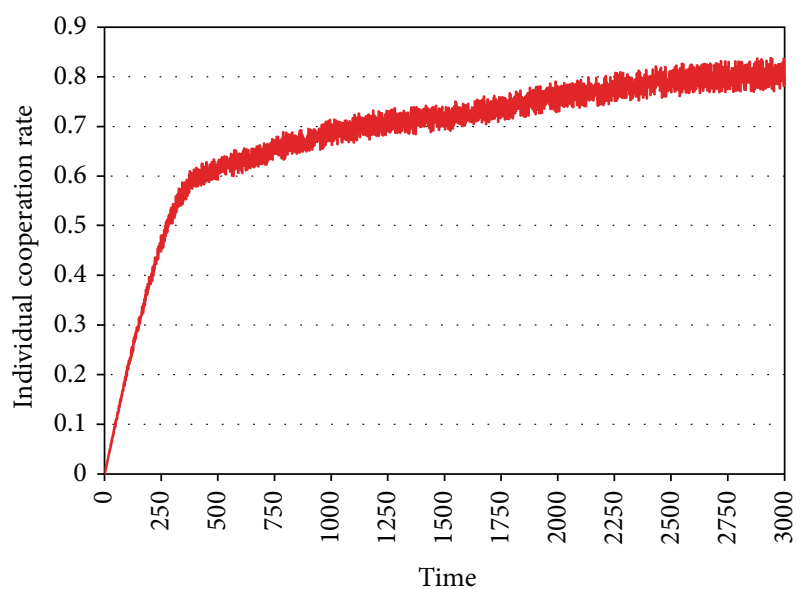

FIGURE 6: Individual cooperation rate of the proposed complex network.

verified to get the Scale-free property to a certain extent. So, many important conclusions of the BA Scale-free model could be applied to the network of regional collaborative innovation conveniently. As we all know, the most notable feature of the Scale-free network is that some highest-degree nodes exist in the networks, which are called "Hub, Hubnode." And they had much more connections that greatly exceed the average. This situation caused the networks to have satisfactory robust stability for failure, but sometimes they might be very delicate, which means that, even more than 80 percent of the nodes are removed or invalidated, and rest ones, if the "Hubs" exist, could still keep the system normal running. Nevertheless, the Hub-nodes themselves suffered a deliberate attack, and the whole network might become paralyzed at once. And the previous research has indicated that there exists significant Matthew effect in the BA Scalefree networks [29]. This makes it easier for the local government to mainly focus on protecting the Hub-nodes at a lower cost and make the best of the dependencies and relationships on these nodes. Meanwhile, another responsibility of the government was that they should try their best to establish

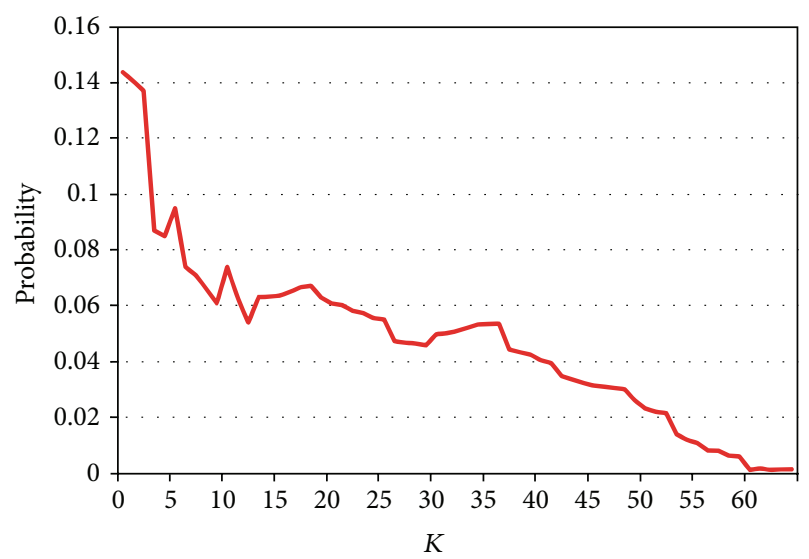

FIGURE 7: Degree distribution of the proposed complex network.

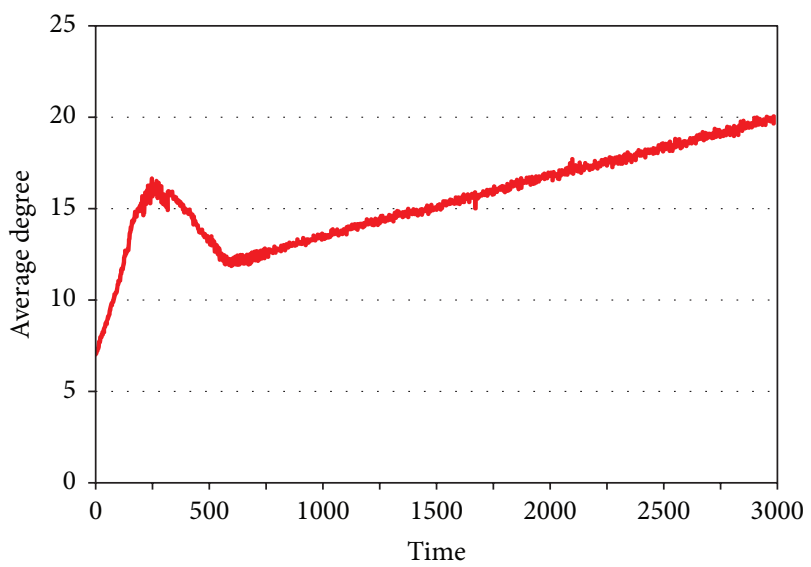

FIGURE 8: Average degree of the proposed complex network.

a balance mechanism to allocate resources in order to prevent the overall development from becoming excess uneven.

Next, we will testify the average degree of the complex network since the network evolution has happened. Experimental results in Figure 8 have shown that the average degree was rising rapidly at first and then decreased. At last, it increased slowly once again. The reasons why it happened in our real life might be as follows. (1) Individuals in the proposed network have established the relationships with broad opportunities to their neighbors, and in the initial stage the rapid growth in the number has played an important role in this framework. (2) When the edges in this network increase to a specific threshold, with limited resources, a substantial part of the participants may discover that there are more and more connections among their neighbors cannot continue to be matched. (3) Afterwards, in the next stage, as communication costs continued slowing down, and the average degree of the networks may reach the steadily rising state again.

At the end of the experiment, in Figure 9, we will test the percentage of neighbors without influencing their individual distribution. This metric was designed to measure the participation levels of the nodes in the system. It has adequately reflected the network evolution to some extent. To facilitate 


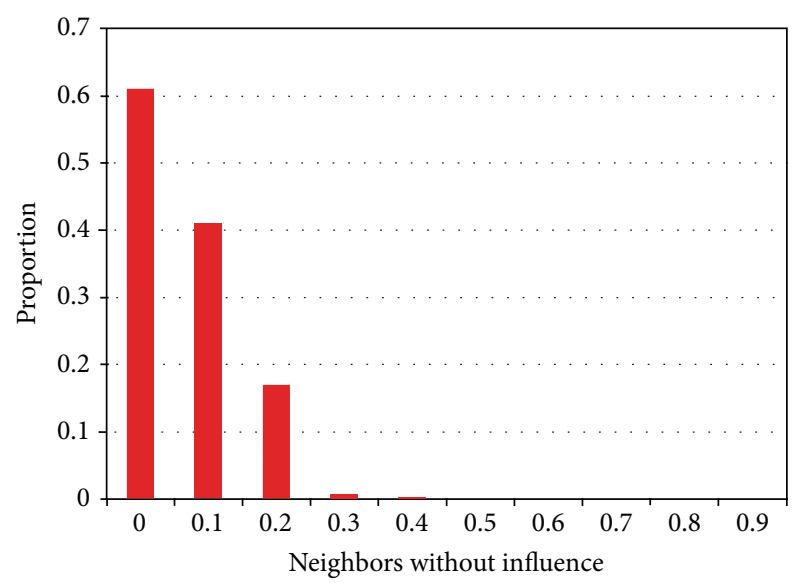

FIGURE 9: Individual neighbor distribution of the proposed complex network.

computations, all associated measures in the histogram have been standardized. Figure 9 vividly demonstrates that the majority neighbors are all effectively changed based on the proposed algorithm, as a continual evolution of the network. As a dynamics algorithm, most of the nodes in the network have joined the evolutionary process. And the proportion of isolated nodes in general are always maintained at a relatively low level. In other words, the histogram in Figure 9 has also verified the efficiency of the proposed method well.

Integrating the above experimental results, several suggestions could be obtained by analyzing the evolution of regional collaborative innovation systems and some known characteristics of the complex networks:

(1) As a representative sample of complex networks, the regional collaborative innovation systems have exhibited distinguishing features of the Small-World effect and Scale-free property. These characteristics strongly correlate with network's robustness. The local governments should pay more attention to the Hub-nodes in order to ensure stability of the whole network at a low cost unless the central node suffered a direct attack deliberately.

(2) We discover the collaborative innovation alliance, no matter indigenous or across geographies, could greatly help to enhance the innovation capacity of the organization members and improve internal structures of the whole networks. In order to continuously increase comprehensive strength of the concrete regional collaborative innovation system, we recommend all related participants should accelerate development by intensified cooperation.

(3) The study found that local governments play a very important role in improving the comprehensive innovative ability. Furthermore, the policy maker should pay more attention to raising new ideas, rolling out effective policies, and promoting the level of cooperation. So, it is also a salutary attempt to make some more operational mechanisms. And the superiority of the method would gradually be embodied through the execution.

\section{Conclusion}

In this paper, we mainly focused on a novel evolutionary algorithm of the regional collaborative innovation. And the organizational structure and capability model was composed of government, universities, research institutes, intermediary organizations, financial institutions, and enterprises. In this framework, there are about five components, such as (1) innovation guaranteed ability, (2) innovation research and development ability, (3) innovation integration ability, (4) innovation transformation ability, and (5) innovation supporting environment. The network architecture has shown common topological properties of complex network, such as Small-World effect and Scale-free property. In the proposed algorithm, we add the condition that a certain node can only be connected to a fixed number of neighbors. Furthermore, the evolution process of regional collaborative innovation system can be represented by conducting simulation experiment and computing the probability density function. Most of the empirical data are collected from the official website of the National Bureau of Statistic of China (NBS) and some other open sources. Finally, experimental results evaluated effectiveness of the proposed algorithm. And the specific structural characteristics of the network topology could make some suggestions to policy maker as a reference at the same time. In the future, we would further expand the proposed works in the following aspects:

(1) Try to make further effectiveness evaluations of the proposed algorithm, and the related datasets may be collected from some other countries.

(2) We can use the evolutionary algorithm to compare the differences among the evolution processes of regional collaborative innovation in diverse locations.

\section{Conflict of Interests}

The authors confirm that this paper content has no conflict of interests.

\section{Acknowledgments}

This research is financially supported by the National Natural Science Foundation of China (Grant no. 71473263) and the Specialized Research Fund for Doctoral Program of Higher Education of China (Grant no. 20134307110020).

\section{References}

[1] M. Melanie, Complexity: A Guided Tour, Oxford University Press, Oxford, UK, 2011.

[2] D. J. Watts and S. H. Strogatz, "Collective dynamics of 'smallworld' networks,” Nature, vol. 393, no. 6684, pp. 440-442, 1998.

[3] A.-L. Barabási and R. Albert, "Emergence of scaling in random networks," Science, vol. 286, no. 5439, pp. 509-512, 1999. 
[4] F. P. Miller, A. F. Vandome, and J. McBrewster, Complex Network, Alphascript Publishing, 2010.

[5] S. V. Buldyrev, R. Parshani, G. Paul, H. E. Stanley, and S. Havlin, "Catastrophic cascade of failures in interdependent networks," Nature, vol. 464, no. 7291, pp. 1025-1028, 2010.

[6] W. Yu, P. DeLellis, G. Chen, M. di Bernardo, and J. Kurths, "Distributed adaptive control of synchronization in complex networks," IEEE Transactions on Automatic Control, vol. 57, no. 8, pp. 2153-2158, 2012.

[7] S. Chen, W. Huang, C. Cattani, and G. Altieri, "Traffic dynamics on complex networks: a survey," Mathematical Problems in Engineering, vol. 2012, Article ID 732698, 23 pages, 2012.

[8] M. E. Newman and D. J. Watts, "Renormalization group analysis of the small-world network model," Physics Letters A, vol. 263, no. 4-6, pp. 341-346, 1999.

[9] A.-L. Barabási, R. Albert, and H. Jeong, "Scale-free characteristics of random networks: the topology of the world-wide web," Physica A: Statistical Mechanics and Its Applications, vol. 281, no. 1, pp. 69-77, 2000.

[10] E. Estrada, N. Hatano, and M. Benzi, "The physics of communicability in complex networks," Physics Reports. A Review Section of Physics Letters, vol. 514, no. 3, pp. 89-119, 2012.

[11] B. T. Asheim and A. Isaksen, "Regional innovation systems: the integration of local 'sticky' and global 'ubiquitous' knowledge," The Journal of Technology Transfer, vol. 27, no. 1, pp. 77-86, 2002.

[12] S. Abe and N. Suzuki, "Dynamical evolution of the community structure of complex earthquake network," Euro Physics Letters, vol. 99, no. 3, Article ID 39001, 2012.

[13] S. Rubach, "Collaborative regional innovation initiatives: a booster for local company innovation processes?" Systemic Practice and Action Research, vol. 26, no. 1, pp. 3-21, 2013.

[14] D. Chen, L. Lü, M.-S. Shang, Y.-C. Zhang, and T. Zhou, "Identifying influential nodes in complex networks," Physica A: Statistical Mechanics and Its Applications, vol. 391, no. 4, pp. 1777-1787, 2012.

[15] D. Kasthurirathna, M. Piraveenan, and M. Harré, "Influence of topology in the evolution of coordination in complex networks under information diffusion constraints," The European Physical Journal B, vol. 87, no. 1, article 3, 15 pages, 2014.

[16] A. Anzo and J. G. Barajas-Ramirez, "Synchronization in complex networks under structural evolution," Journal of the Franklin Institute. Engineering and Applied Mathematics, vol. 351, no. 1, pp. 358-372, 2014.

[17] R. Alvarez-Martinez, G. Cocho, R. F. Rodríguez, and G. Martínez-Mekler, "Birth and death master equation for the evolution of complex networks," Physica A: Statistical Mechanics and Its Applications, vol. 402, pp. 198-208, 2014.

[18] G. A. Pagani and M. Aiello, "Power grid complex network evolutions for the smart grid," Physica A: Statistical Mechanics and Its Applications, vol. 396, pp. 248-266, 2014.

[19] L. Wang, P. Yu, Z. Wang, C. Yang, and Q. Ye, "On the evolution of Linux kernels: a complex network perspective," Journal of software: Evolution and Process, vol. 25, no. 5, pp. 439-458, 2013.

[20] D.-L. Duan and R.-J. Zhan, "Evolution mechanism of node importance based on the information about cascading failures in complex networks," Acta Physica Sinica, vol. 63, no. 6, Article ID 068902, 2014.

[21] L. Huang, W. Wang, and M. Wang, "Simulation research of space-time evolution of emergency logistics network reliability based on complex network theory," Discrete Dynamics in Nature and Society, vol. 2013, Article ID 303187, 7 pages, 2013.
[22] J. Leskovec, J. Kleinberg, and C. Faloutsos, "Graph evolution: densification and shrinking diameters," ACM Transactions on Knowledge Discovery from Data, vol. 1, no. 1, article 2, 2007.

[23] T. Hadzibeganovic, F. W. S. Lima, and D. Stauffer, "Evolution of tag-mediated altruistic behavior in one-shot encounters on large-scale complex networks," Computer Physics Communications, vol. 183, no. 11, pp. 2315-2321, 2012.

[24] A. Herz and C. Olivier, "Transnational social network analysis," Transnational Social Review, vol. 2, no. 1, pp. 11-29, 2012.

[25] J. Kim and T. Wilhelm, "What is a complex graph?" Physica A: Statistical Mechanics and its Applications, vol. 387, no. 11, pp. 2637-2652, 2008.

[26] 2015 (Chinese), http://www.stats.gov.cn/tjsj/ndsj/.

[27] M. C. Rios, C. R. McConnell, and S. L. Brue, Economics: Principles, Problems, and Policies, McGraw-Hill, 2013.

[28] D. H. Meadows, D. L. Meadows, J. Randers, and W. W. Behrens, The Limits to Growth, vol. 102, New American Library, New York, NY, USA, 1972.

[29] H. A. Che and J. F. Gu, "Scale free networks and their significance for systems science," Systems Engineering-Theory \& Practice, vol. 24, no. 4, pp. 11-16, 2004 (Chinese). 


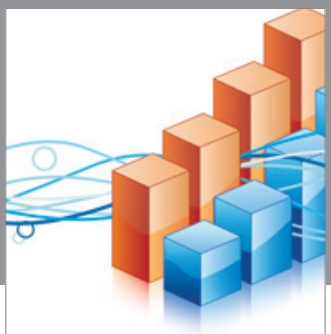

Advances in

Operations Research

vatem alat4

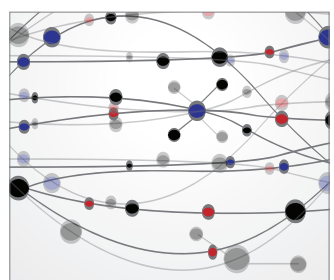

\section{The Scientific} World Journal
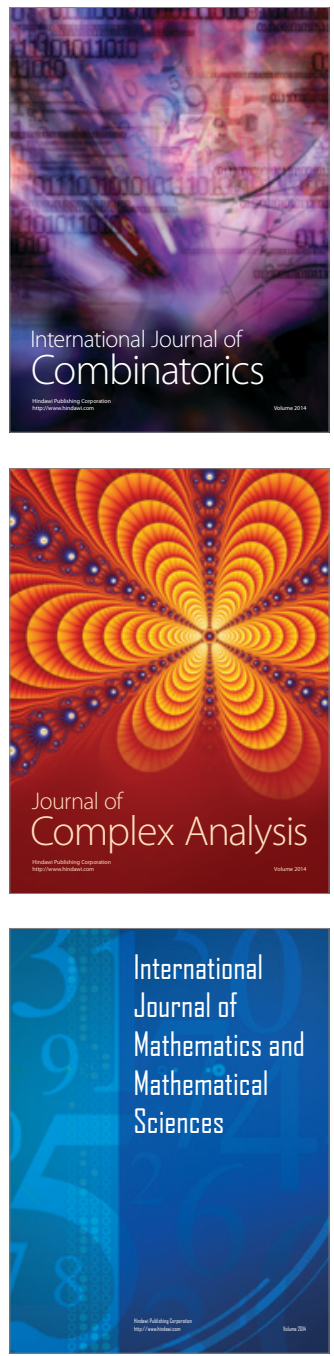
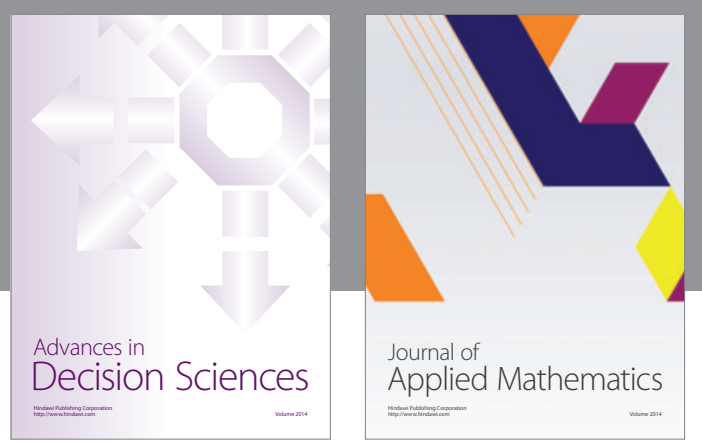

Algebra

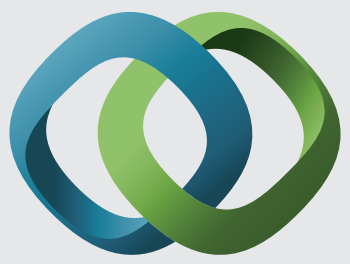

\section{Hindawi}

Submit your manuscripts at

http://www.hindawi.com
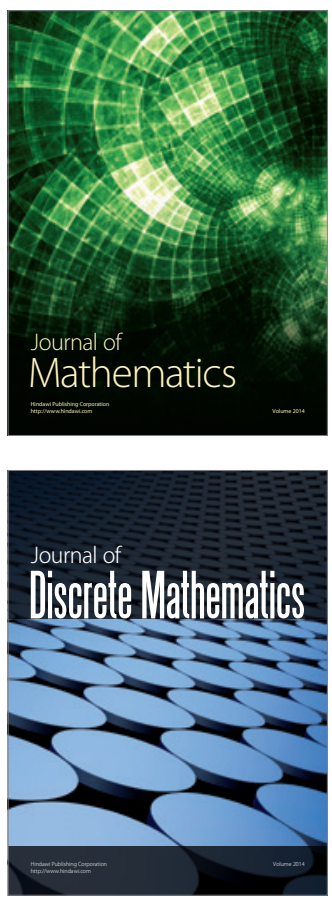

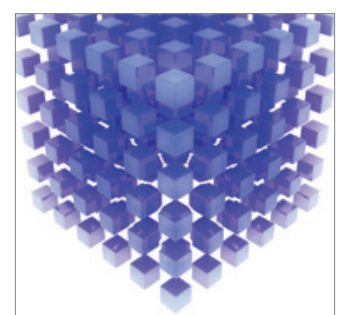

Mathematical Problems in Engineering
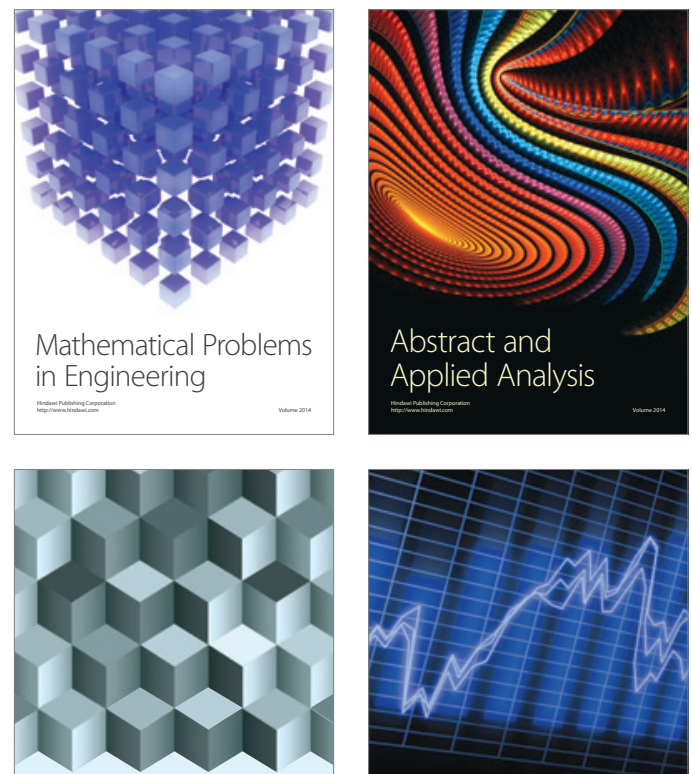

Journal of

Function Spaces

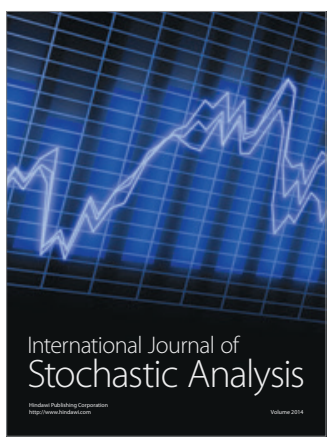

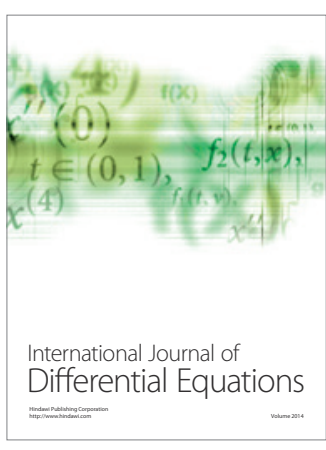
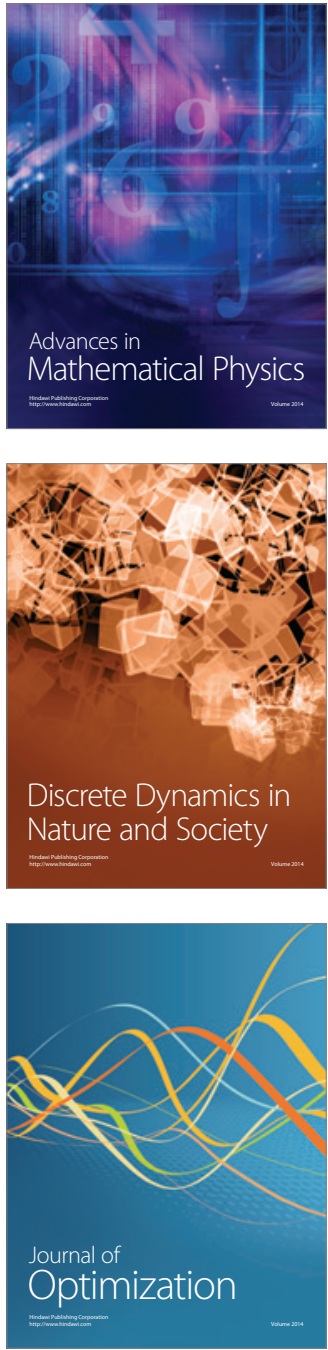